\title{
Quartets of superdeformed bands and supersymmetry breaking
}

\author{
R.D. Amado $^{(1),(2)}$, R. Bijker ${ }^{(3)}$, F. Cannata(1),(4), \\ J.P. Dedonder ${ }^{(1),(5)}$ and N.R. Walet ${ }^{(2)}$ \\ (1) Paul Scherrer Institute, CH-5232 Villigen-PSI, Switzerland \\ (2) Department of Physics, University of Pennsylvania, Philadelphia, PA 19104, USA ${ }^{(a)}$ \\ (3) R.J. Van de Graaff Laboratory, University of Utrecht, P.O. Box 80000, NL-3508 TA Utrecht, \\ The Netherlands \\ (4) Dipartimento di Fisica and INFN, I-40126 Bologna, Italy ${ }^{(b)}$ \\ (5) Laboratoire de Physique Nucléaire, Université Paris 7, 2 Place Jussieu, F-75251 Paris Cedex \\ 05 and Division de Physique Théorique, IPN, F-91406 Orsay, France ${ }^{(c)}$
}

We examine the prediction of supersymmetric quantum mechanics that bands with identical gamma-ray energies occur in quartets. The experimental data suggest that this scenario is actually realized in nature. In the $A=150$ mass region, four known pairs of isospectral bands can be grouped in two quartets, while there are indications of such patterns around $A=190$. We introduce a small supersymmetry breaking, necessary to describe the details of the data. We derive relations among the transition rates that can be used to test our predictions.

21.10.Re, 11.30.Pb, 21.60.Fw, 23.20.Lv

Typeset Using REVTEX 


\section{INTRODUCTION}

In a recent letter [1] we showed that the remarkable identical gamma ray bands (isospectral gamma rays) seen in neighboring superdeformed nuclei [2,3] have a natural description in terms of supersymmetric quantum mechanics (SSQM). The role of supersymmetry in superdeformed nuclei has not in general been emphasized except in «1. The SSQM picture requires that bands with isospectral gamma rays in an even and neighboring odd nucleus be accompanied by two more isospectral bands, one in the odd nucleus and another in a neighboring even nucleus. As we have shown in [牦] this is most naturally realized when the even nuclei differ by two nucleons. We also suggested that two of the bands actually occurred at a very high excitation energy, so that they would be unobservable in experiment. In this paper we point out that all four band might actually be observable. We show that the experimentally observed isospectral pairs can very naturally be grouped together in two quartets. We point out that two such quartets of gamma ray sequences have indeed been observed in superdeformed nuclei in the $A=150$ mass region, although to our knowledge they have seldom been associated together [5,6]. As could be expected, the quartets are not perfectly isospectral which suggests a small breaking of the supersymmetry.

In this paper we devise a simple form of supersymmetry breaking and show that this accounts for the bulk of the experimental data related to these quartets. Our work on symmetry breaking gives promise of making a connection between our approach and more microscopic ones. Because SSQM relates the four gamma ray cascades that make up the quartet, it also makes predictions about transition rates in the four bands. These are presented here to stimulate experimental interest when the new facilities to study superdeformed nuclei come on line [7].

\section{GAMMA RAY QUARTETS IN SUPERDEFORMED NUCLEI}

The recently observed sequences of isospectral gamma rays in neighboring even and odd nuclei lead naturally to an interpretation in terms of supersymmetric quantum mechanics. As is well known SSQM is designed to have equal energy eigenvalues for bosonic (even nucleus) and fermionic (odd nucleus) states. In addition SSQM requires that the dimension of the bosonic part and the fermionic part of a given degenerate multiplet be identical. For the case of superdeformed nuclei this implies that for a given excitation energy the number of states in the even nucleus equals that in the neighboring odd nucleus. If the state in the even nucleus has angular momentum $L$ (integer) and the corresponding state in the odd nucleus $J$ (half integer), the degeneracies are $2 L+1$ and $2 J+1$, respectively. Of course these two numbers can never be equal. In [1] we showed that the state counting problem can be solved in the following way. Suppose the state in the odd nucleus is obtained by coupling one particle (or hole) with spin or pseudospin 8$] s=\frac{1}{2}$ to the corresponding state in the even nucleus. The angular momenta in the odd system are then $J_{>}=L+\frac{1}{2}$ and $J_{<}=L-\frac{1}{2}$. Suppose further that in the even nucleus with two particles (or two holes) more than in the original even nucleus there is a level with angular momentum $L$. Now there are $2(2 L+1)$ even states and precisely the same number of odd states. These four states form a degenerate SSQM quartet. Transitions between sets of these states will lead to four isospectral gamma ray sequences, one in the even nucleus $A$, one in the other even nucleus 
$A \pm 2$, and two in the odd $A \pm 1$ nucleus. One of these last two is among the $J_{>}$states and the other among the $J_{<}$states. As we shall show below cross-over transitions between $J_{<}$ and $J_{>}$are very small.

It is this quartet of gamma rays sequences that is the hallmark of SSQM. Let us examine two candidates. In Tables I and II we show that all four known isospectral pairs can be grouped in two of these quartets. In Table I we compare gamma ray sequences in four superdeformed bands in the adjacent nuclei ${ }^{152} \mathrm{Dy},{ }^{151} \mathrm{~Tb}$ (twice) and ${ }^{150} \mathrm{Gd}$ [2, 9, 10,11, 12]. As is well known spins have not been measured yet, and we have thus chosen spin assignments consistent with a SSQM interpretation. We have followed the spin assignments in the compilation [12] as closely as possible. We see that the four bands are very close in energy, but are not completely degenerate. In spite of the breaking of supersymmetry it is clear that the quartet bears the strong family resemblance suggested by SSQM. A second example of such a quartet is shown in Table II . Here the four bands are in ${ }^{148} \mathrm{Gd},{ }^{147} \mathrm{Gd}$ (twice), and ${ }^{146} \mathrm{Gd}$ [5, 6, 12, 13, 14]. Again the spins have been assigned to stress the SSQM connection, and again we see the general pattern of slightly broken SSQM. The pattern does seem to shift around $L=46$, which is due to a level crossing that is not contained in the simple SSQM quartet picture. The crossing occurs in two bands that do not form an isospectral pair: the 1b-band in ${ }^{146} \mathrm{Gd}$ and the $1 \mathrm{~b}$-band in ${ }^{147} \mathrm{Gd}$. Before the level crossing the last band is isospectral with the $1 \mathrm{~b}$-band in ${ }^{148} \mathrm{Gd}$, and the first with the $2 \mathrm{~b}$-band in ${ }^{147} \mathrm{Gd}$. As can be seen from Fig. 2 the nature of the crossing is very similar in both cases. Thus the band crossing stresses the correlation between the two isospectral pairs within the quartet. It suggests that a description in terms of two independent pairs of superdeformed bands is not adequate.

It would certainly be interesting to pursue the search for identical superdeformed bands [15] and investigate whether more supersymmetric quartets exist. There are preliminary indications that such quartets are present in the $A=190$ mass region (see Table III). Furthermore, it is very important to determine the spins experimentally. The spin assignments of SSQM are a central feature of the theory and their verification or refutation is crucial to test the application of supersymmetry to superdeformed nuclei. Note that the SSQM spin assignments do not always agree with other phenomenological determinations [16, 17].

\section{SSQM BREAKING}

In a SSQM description the quartet of states discussed in Section II is degenerate. This degeneracy implies that the energy only depends on $L$. A small spin-orbit splitting will break the supersymmetry. At the algebraic level of our treatment it is irrelevant whether this is a spin-orbit or a pseudo-spin-orbit coupling. For each nucleus, we take a Hamiltonian of the form

$$
H_{i}=a_{i} \mathbf{L} \cdot \mathbf{L}+b \mathbf{s} \cdot \mathbf{L},
$$

where $\mathbf{L}$ is the angular momentum of the core and $\mathbf{s}$ is the spin (or pseudo-spin) of the odd particle (hole). The coefficients $a_{i}$ are inversely proportional to the moment of inertia in each nucleus. The strength of the spin-orbit coupling, $b$, is taken the same for each nucleus. In the sequences we are considering (Tables I and II) we label the three nuclei by $i=0$ for ${ }^{152} \mathrm{Dy}$ or ${ }^{148} \mathrm{Gd}$, by $i=1$ for ${ }^{151} \mathrm{~Tb}$ or ${ }^{147} \mathrm{Gd}$ and by $i=2$ for ${ }^{150} \mathrm{Gd}$ or ${ }^{146} \mathrm{Gd}$. 
Supersymmetry implies that all the $a_{i}$ 's are equal. A plot of the gamma ray energies (see Figures I and II) against the core angular-momentum $L$ confirms this by revealing a near perfect straight line with almost the same slope for all transitions. With $a_{0}$ as the reference, we assume small $L$ dependent departures in $a_{1}$ which we parametrize as $a_{1}=a_{0}+\frac{\epsilon}{L}$.18. If this departure is due to the extra hole (or extra particle), we expect $a_{2}=a_{0}+\frac{2 \epsilon}{L}$. With these expressions we find for the transition energies $E_{\gamma i}(I)=E_{i}(I)-E_{i}(I-2)$, where $E_{i}(I)$ is the excitation energy of the state with angular momentum $I$,

$$
\begin{aligned}
E_{\gamma 0}(L) & =a_{0}(4 L-2), \\
E_{\gamma 1}\left(L \pm \frac{1}{2}\right) & =a_{0}(4 L-2)+2 \epsilon \pm b, \\
E_{\gamma 2}(L) & =a_{0}(4 L-2)+4 \epsilon .
\end{aligned}
$$

In this parametrization

$$
\begin{aligned}
\Delta E_{\gamma 01} & =E_{\gamma 0}(L)-E_{\gamma 1}\left(L+\frac{1}{2}\right)=\Delta E_{\gamma 12} \\
& =E_{\gamma 1}\left(L-\frac{1}{2}\right)-E_{\gamma 2}(L)=2 \epsilon+b .
\end{aligned}
$$

Both in the case of Table I and Table II this difference is essentially zero. This relationship among the gamma ray sequences in the Gadolinium isotopes has been noted by Ragnarsson [6] but not in the context of SSQM and with different spin assignments. (We stop the comparison at $L=46$ in Table II as discussed in the previous Section). We have no simple explanation for why $E_{\gamma 1}\left(L+\frac{1}{2}\right)=E_{\gamma 0}(L)$, but we see that for both Tables our parametrization immediately gives that if $E_{\gamma 1}\left(L+\frac{1}{2}\right)=E_{\gamma 0}(L)$, then $E_{\gamma 2}(L)=E_{\gamma 1}\left(L-\frac{1}{2}\right)$ as seen. The parametrization also implies that $E_{\gamma 2}(L)-E_{\gamma 0}(L)=4 \epsilon$ independent of $L$ and that too is fairly well born out in the Tables. We find for the nuclei of Table I, $\epsilon=-b / 2=$ $-6.3 \pm 1.3 \mathrm{keV}$, which should be compared to $a_{0}=47 \pm 1 \mathrm{keV}$. Thus the term proportional to $\epsilon$ is indeed a small perturbation in the expression for $a_{2}$. Similarly, for Table II we find $\epsilon=-b / 2=8.3 \pm 0.6 \mathrm{keV}$ and $a_{0}=55 \pm 3$.

We see that a very simple approach to SSQM breaking based on a weak spin-orbit coupling and a slight difference in how moments of inertia change with added particles or holes accounts for the data of Tables I and II. Although at the moment we do not have a microscopic understanding of the pair wise equality of gamma ray energies, $E_{\gamma 1}(L+$ $\left.\frac{1}{2}\right)=E_{\gamma 0}(L)$ and $E_{\gamma 1}\left(L-\frac{1}{2}\right)=E_{\gamma 2}(L)$, we have shown a mechanism in terms of a broken supersymmetry that exhibits this pattern. Whether the remaining degeneracy is accidental or the result of a deeper symmetry remains to be seen. It is our goal in this paper to show that we can realize the pattern of super-symmetry breaking apparent in the experimental data.

\section{TRANSITION STRENGTHS}

In the tentative spin assignments in Table I and II we have assumed that the subsequent states in a superdeformed band differ by two units of angular momentum. If we further adopt a pseudo-spin picture in which the $E 2$ transition operator is independent of the pseudospin, the ratio between the four possible intraband transition probabilities only depends on geometric factors from angular momentum algebra [19]. Again taking the $B(E 2)$ value in the $A$ nucleus as a reference, we find the following relations for the intraband transitions 


$$
\begin{aligned}
B\left(E 2 ; J_{>}=L+\frac{5}{2} \rightarrow J_{>}=L+\frac{1}{2}\right)= & B(E 2 ; L+2 \rightarrow L) \\
B\left(E 2 ; J_{<}=L+\frac{3}{2} \rightarrow J_{<}=L-\frac{1}{2}\right)= & \frac{L(2 L+5)}{(2 L+1)(L+2)} \\
& \times B(E 2 ; L+2 \rightarrow L)
\end{aligned}
$$

The transition probabilities in the even nuclei in the quartet are identical in this scheme. For typical values of the angular momenta in superdeformed bands (see Tables I and II) the intraband transitions energies are nearly the same (isospectral). The related interband transition between the two bands in the odd nucleus,

$$
\begin{aligned}
B\left(E 2 ; J_{<}=L+\frac{3}{2} \rightarrow J_{>}=L+\frac{1}{2}\right)= & \frac{2}{(2 L+1)(L+2)} \\
& \times B(E 2 ; L+2 \rightarrow L),
\end{aligned}
$$

is highly suppressed with respect to the intraband transitions for typical values of the angular momentum. The other interband transition from $J_{>}=L+\frac{5}{2}$ to $J_{<}=L-\frac{1}{2}$ is strictly forbidden for quadrupole radiation.

Following the notation of the previous section we label the bands by $i=0,1 \pm, 2$. The ratio of the transition probabilities $B_{i j}$ only depends on a simple geometric factor $B_{00}$ : $B_{22}: B_{1+1+}: B_{1-1-}: B_{1-1+}=1: 1: 1: \frac{L(2 L+5)}{(2 L+1)(L+2)}: \frac{2}{(2 L+1)(L+2)}$. For typical values of $L$ in superdeformed bands it is appropriate to take the large $L$ limit for these ratios, giving $1: 1: 1: 1: \frac{1}{L^{2}}$, which shows that for large $L$ all intraband transition probabilities are equal and that the interband transition from $J_{<}=L+\frac{3}{2}$ to $J_{>}=L+\frac{1}{2}$ is down by $\frac{1}{L^{2}}$, a very big suppression.

The other interband transition from $J_{>}=L+\frac{1}{2}$ to $J_{<}=L-\frac{1}{2}$ depends on the same matrix element as the static quadrupole moments. With the quadrupole moment $Q_{0}(L)$ in the $A$ nucleus as a reference we find

$$
\begin{aligned}
& Q_{1}\left(L+\frac{1}{2}\right)=Q_{0}(L) \\
& Q_{1}\left(L-\frac{1}{2}\right)=\frac{(L-1)(2 L+3)}{L(2 L+1)} Q_{0}(L) .
\end{aligned}
$$

The ratio of the quadrupole moments of the states in the four superdeformed bands is then $Q_{0}(L): Q_{2}(L): Q_{1}\left(L+\frac{1}{2}\right): Q_{1}\left(L-\frac{1}{2}\right)=1: 1: 1: \frac{(L-1)(2 L+3)}{L(2 L+1)}$, which shows that in the large $L$ limit the quadrupole moments of the members of a quartet are identical. The E2 transition between the two states in the odd nucleus belonging to the same quartet is suppressed by $1 / L^{2}$,

$$
B\left(E 2 ; J_{>}=L+\frac{1}{2} \rightarrow J_{<}=L-\frac{1}{2}\right)=\frac{3}{(2 L+1)(L+1)} B(E 2 ; L \rightarrow L) .
$$

The corresponding gamma ray energy for this transition is determined by the spin-orbit splitting, $E_{\gamma}=\frac{1}{2} b(2 L+1)$. The present data on lifetimes and quadrupole moments for superdeformed bands is still quite scarce [13, 14, 20,21, 10] and it will be certainly interesting to check the above predictions when the technology develops to the point of accurate measurements of lifetimes. 
We stress that our predictions do not depend on the details of the dynamics, but only on the SSQM prediction that the four states have nearly identical internal structures and therefore have the same reduced matrix elements for quadrupole transitions. They further depend on the assumption that the odd states are constructed by coupling a spin (or pseudospin) $s=\frac{1}{2}$ to the orbital state. The symmetry breaking considered in the previous section is diagonal and does not affect the transition strengths. In spite of the weak nature of all these assumptions the connection of quartets across even and odd nuclei makes interesting and verifiable predictions.

These prediction should hold to high accuracy, and should not be confused with the much weaker relations that can be derived in the Bohr-Mottelson model for nuclei with roughly equal deformation.

\section{PROSPECTS AND SUMMARY}

Supersymmetric quantum mechanics relates states in odd and even neighboring nuclei. It describes a quartet of states across nuclei with $A$ (even) nucleons, $(A+1)$ and $(A+2)$ nucleons that are degenerate and hence lead to four isospectral bands in the nuclei, one each in the $A$ and $(A+2)$ nuclei and two in the $(A+1)$ nucleus. Although, we pointed out [1] that other realizations of SSQM are possible, it seems that the $A,(A+1),(A+2)$ scheme is not only the most natural, but is actually realized in nature. In this paper we have shown that two examples of such quartets appear to exist in the data although they have seldom been regrouped to emphasize the SSQM connection. It may very well be that these quartets of gamma ray sequences in superdeformed nuclei are the best example of supersymmetry so far discovered in physics.

We have tried to realize the breaking of supersymmetry in the simplest possible way, in order to describe the experimental data. We have not attempted to derive the symmetry from an underlying microscopic Hamiltonian. Such an approach would be complementary to our approach, but it seems highly unlikely that current mean-field techniques can give the required accuracy.

Our approach makes definite assumptions about the spins of the related states and predicts equal transition strengths across the quartets. New experiments with EUROBALL and GAMMASPHERE could be very helpful in checking the spin assignments and the predictions for the transition strengths, as well as in discovering possible new examples of quartets of superdeformed bands. As we have already stated, there are indications of such quartets in the $A=190$ mass region. One can look either for isotopic or isotonic quartets. The presently available data [12] suggests two examples. The better of the two is an isotopic $Z=80$ quartet composed of the superdeformed band in ${ }^{192} \mathrm{Hg}$ [22], two of the four known bands $\left({ }^{193} \mathrm{Hg}(2)\right.$ and ${ }^{193} \mathrm{Hg}(3)$, [23]) in ${ }^{193} \mathrm{Hg}$ and one of the three known bands $\left({ }^{194} \mathrm{Hg}(3)\right.$, [24, 25, 26]) in ${ }^{194} \mathrm{Hg}$. This is displayed in Table III where the equality between the quantities $\Delta E_{\gamma 01}$ and $\Delta E_{\gamma 12}$ holds within the experimental uncertainties. We obtain $\epsilon=-0.05 \pm 0.48$ $\mathrm{keV}$ and $b=8.85 \pm 2.35 \mathrm{keV}$. Note also that by shifting the spin of the last two bands up by two units, the pattern remains the same but the values of the symmetry breaking parameters $\epsilon$ and $b$ are changed to $9.3 \pm 0.42 \mathrm{keV}$ and $-9.85 \pm 2.19 \mathrm{keV}$, respectively. An other possibility may be the isotonic $N=112$ quartet made of ${ }^{192} \mathrm{Hg}$ [22], ${ }^{193} \mathrm{Tl}(\mathrm{a}),{ }^{193} \mathrm{Tl}(\mathrm{b})$ [27] and ${ }^{194} \mathrm{~Pb}$ [28] though, there, the difference between $\Delta E_{\gamma 01}$ and $\Delta E_{\gamma 12}$ is beyond experimental errors and 
cannot be accommodated by the simple SSQM breaking scheme of Section III. However the measured quadrupole moments [20,21, 10] for the superdeformed bands in ${ }^{192} \mathrm{Hg}$ and ${ }^{194} \mathrm{~Pb}$ agree within experimental errors. The balance of boson and fermion degrees of freedom, which was discussed in Section II and in [1], implies that these two examples which involve the same band in the ${ }^{192} \mathrm{Hg}$ nucleus should be incompatible unless there are two (almost) degenerate bands in ${ }^{192} \mathrm{Hg}$ or unlesss the supersymmetry multiplet is otherwise enlarged. At the present time only one superdeformed band has been observed in ${ }^{192} \mathrm{Hg}$ [22]. Further investigations around mass 192 would be very valuable to clarify these points.

To summarize, our SSQM description is not cast in terms of microscopic variables. Some of the dynamics comes in via the symmetry breaking. The quartet multiplets are not perfectly isospectral. Most of this symmetry breaking can be accounted for by a small (pseudo)spin orbit term and by some very simple $L$-dependent change in the moments of inertia. These correction terms should begin to give some insight into the connection between SSQM dynamics and the more conventional dynamical avenues [29,30,26.

\section{ACKNOWLEDGMENTS}

RDA, FC, and JPD would like to thank the Paul Scherrer Institute and Milan Locher for bringing us together and for providing a very pleasant environment in which much of this work was done. RDA and NRW are supported in part by the U.S. National Science Foundation and RB by the Stichting voor Fundamenteel Onderzoek der Materie (FOM) with financial support from the Nederlandse Organisatie voor Wetenschappelijk Onderzoek (NWO). The Division de Physique Théorique is a Research Unit of the Universities Paris 11 and 6 associated to CNRS. 


\section{REFERENCES}

${ }^{a}$ Permanent address of RDA.

${ }^{b}$ Permanent address of FC.

${ }^{c}$ Permanent addresses of JPD.

[1] R. D. Amado, R. Bijker, F. Cannata and J.-P. Dedonder, Phys. Rev. Lett. 67, 2777 (1991).

[2] T. Byrski et al., Phys. Rev. Lett. 64, 1650 (1990).

[3] P. J. Twin, Proc. Conf on Nuclear Structure in the Nineties (Oak Ridge, April 1990), Nucl. Phys. A520, 17c (1990);

J. F. Scharpey-Schafer, Prog. Part. Nucl. Phys., 28, 187 (1992).

[4] A. Gelberg, P. von Brentano and R. F. Casten, J. Phys. G: Nucl. Part. Phys. 16, L143 (1990).

[5] K. Zuber et al, Phys. Lett. B254, 308 (1991).

[6] I. Ragnarsson, Phys. Lett. B264, 5 (1991).

[7] F. A. Beck, Prog. Part. Nucl. Phys. 28, 443 (1992); I-Yang Lee, ibid., p. 473.

[8] K. T. Hecht and A. Adler, Nucl. Phys. A137, 129 (1969);

A. Arima, M. Harvey and K. Shimizu, Phys. Lett. 30B, 517 (1969)

[9] P. J. Twin et al, Phys. Rev. Lett. 57, 84 (1986).

[10] M. A. Bentley et al, Phys. Rev. Lett. 59, 2141 (1987).

[11] P. Fallon et al, Phys. Lett. B218, 137 (1989).

[12] X.-L. Han and C.-L. Wu, Nuclear Superdeformation Data Tables, to appear in Atomic Data and Nuclear Data Tables 52, (1992).

[13] G. Hebbinghaus et al, Phys. Lett. B240, 311 (1990).

[14] M. A. Deleplanque, C. Beausang, J. Burde, R. M. Diamond, J. E. Draper, C. Duyar, A. O. Macchiavelli, R. J. McDonald, and F. S. Stephens, Phys. Rev. Lett. 60, 1626 (1988).

[15] I. Ahmad, M. P. Carpenter, R. R. Chasman, R. V. F. Janssens, and T. L. Khoo, Phys. Rev. C44, 1204 (1991);

R. F. Casten, N. V. Zamfir, P. von Brentano, and W.-T. Chou, Phys. Rev. C45, R1413 (1992).

[16] J. Y. Zeng, J. Meng, C. S. Wu, E. G. Zhao, Z. Xing, and X. Q. Chen, Phys. Rev. C44, R1745 (1991).

C. S. Wu, J. Y. Zeng, X. Q. Chen, and J. Meng, Phys. Rev. C45, 261 (1992).

[17] J. E. Draper et al, Phys. Rev. C42, R1791 (1990).

[18] M. A. J. Mariscotti, G. Scharff-Goldhaber and B. Buck, Phys. Rev. 178, 1864 (1969);

G. Scharff-Goldhaber, C. B. Dover and A. L. Goldhaber, Annu. Rev. Nucl. Sci. 26, 239 (1976).

[19] R. Bijker and V. K. B. Kota, Ann. Phys. (N. Y.) 187, 148 (1988).

[20] E. F. Moore et al, Phys. Rev. Lett. 64, 3127 (1990).

[21] P. Willsau et al., Prog. Part. Nucl. Phys. 28, 423 (1992).

[22] J. A. Becker, N. Roy, E. A. Henry, M. A. Deleplanque, C. W. Beausang, R. M. Diamond, J. E. Draper, F. S. Stephens, J. A. Cizewski, and M. J. Brinkman, Phys. Rev. C41, R9 (1990);

D. Ye et al, ibid., R13.

[23] D. M. Cullen et al, Nucl. Phys. A520, 105c (1990).

[24] M. A. Riley et al, Nucl. Phys. A512, 178 (1990). 
[25] C. W. Beausang, E. A. Henry, J. A. Becker, N. Roy, S. W. Yates, Z. Phys. A335, 325 (1990).

[26] F. S. Stephens et al, Phys. Rev. Lett. 64, 2623 (1990).

[27] P. B. Fernandez et al, Nucl. Phys. A517, 386 (1990).

[28] K. Theine et al, Z. Phys. A336, 113 (1990);

M. J. Brinkman et al, ibid., p. 115.

[29] R. V. F. Janssens and T. L. Khoo, Annu. Rev. Part. Nucl. Sci. 41, 321 (1991).

J. Dudek, Prog. Part. Nucl. Phys. 28, 131 (1992); ibid. 65, 301 (1991);

W. Nazarewicz and J. Dobaczewski, Phys. Rev. Lett. 68, 154 (1992).

[30] F. S. Stephens, Nucl. Phys. A520, 91c (1990);

F. S. Stephens et al, 


\section{FIGURES}

FIG. 1. Gamma ray energies versus the core angular momentum $L$ for the quartet of superdeformed bands in ${ }^{152} \mathrm{Dy}$ (solid), ${ }^{151} \mathrm{~Tb}(2 \mathrm{~b})$ (dashed) and (1b) (dotted), and ${ }^{150} \mathrm{Gd}(2 \mathrm{~b}$ ) (dashed-dotted). On the scale of the figure the first two and the last two are indistinguishable.

FIG. 2. Gamma ray energies versus the core angular momentum $L$ for the quartet of superdeformed bands in ${ }^{148} \mathrm{Gd}(1 \mathrm{~b})$ (solid), ${ }^{147} \mathrm{Gd}(1 \mathrm{~b})$ (dashed) and (2b) (dotted), and ${ }^{146} \mathrm{Gd}(1 \mathrm{~b})$ (dashed-dotted). 


\section{TABLES}

TABLE I. Quartet of superdeformed bands in ${ }^{150} \mathrm{Gd},{ }^{151} \mathrm{~Tb}$ and ${ }^{152} \mathrm{Dy}$. Spin assignments are from [12], except for a shift of two units of angular momentum in the bands ${ }^{151} \mathrm{~Tb}(1 \mathrm{~b})$ and ${ }^{150} \mathrm{Gd}(2 \mathrm{~b})$. All energies are in $\mathrm{keV}$.

\begin{tabular}{|c|c|c|c|c|c|c|c|c|c|c|}
\hline \multicolumn{2}{|c|}{${ }^{152} \mathrm{Dy}$} & \multicolumn{2}{|c|}{${ }^{151} \mathrm{~Tb}(2 \mathrm{~b})$} & \multicolumn{3}{|c|}{${ }^{151} \mathrm{~Tb}(1 \mathrm{~b})$} & \multicolumn{2}{|c|}{${ }^{150} \mathrm{Gd}(2 \mathrm{~b})$} & \multirow[b]{2}{*}{$\Delta E_{\gamma 12}$} & \multirow[b]{2}{*}{$\Delta E_{\gamma 20}$} \\
\hline $\bar{L}$ & $E_{\gamma 0}$ & $J$ & $E_{\gamma 1}$ & $\Delta E_{\gamma 01}$ & $J$ & $E_{\gamma 1}$ & $L$ & $E_{\gamma 2}$ & & \\
\hline$\overline{24}$ & 602.3 & & & & & & & & & \\
\hline 26 & 647.2 & 26.5 & 647 & 0.2 & & & & & & \\
\hline 28 & 692.2 & 28.5 & 692 & 0.2 & & & & & & \\
\hline 30 & 737.5 & 30.5 & 738 & -0.5 & 29.5 & 728.0 & & & & \\
\hline 32 & 783.5 & 32.5 & 783 & 0.5 & 31.5 & 769.2 & 32 & 770 & -0.8 & -13.5 \\
\hline 34 & 829.2 & 34.5 & 828 & 1.2 & 33.5 & 811.3 & 34 & 813 & -1.7 & -16.2 \\
\hline 36 & 876.1 & 36.5 & 876 & 0.1 & 35.5 & 854.0 & 36 & 856 & -2.0 & -20.1 \\
\hline 38 & 923.1 & 38.5 & 922 & 1.1 & 37.5 & 898.0 & 38 & 900 & -2.0 & -23.1 \\
\hline 40 & 970.0 & 40.5 & 970 & 0.0 & 39.5 & 942.8 & 40 & 944 & -1.2 & -26.0 \\
\hline 42 & 1017.0 & 42.5 & 1016 & 1.0 & 41.5 & 988.7 & 42 & 990 & -1.3 & -27.0 \\
\hline 44 & 1064.8 & 44.5 & 1063 & 1.8 & 43.5 & 1034.9 & 44 & 1036 & -1.1 & -28.8 \\
\hline 46 & 1112.7 & 46.5 & 1112 & 0.7 & 45.5 & 1082.5 & 46 & 1082 & 0.5 & -30.7 \\
\hline 48 & 1160.8 & 48.5 & 1158 & 2.8 & 47.5 & 1130.2 & 48 & 1130 & 0.2 & -30.8 \\
\hline 50 & 1208.7 & 50.5 & 1207 & 1.7 & 49.5 & 1178.9 & 50 & 1180 & -1.1 & -28.7 \\
\hline 52 & 1256.6 & 52.5 & 1256 & 0.6 & 51.5 & 1228.5 & 52 & 1229 & -0.5 & -27.6 \\
\hline 54 & 1304.7 & 54.5 & 1305 & -0.3 & 53.5 & 1278.5 & 54 & 1277 & 1.5 & -27.7 \\
\hline 56 & 1353.0 & 56.5 & 1353 & 0.0 & 55.5 & 1330.0 & 56 & 1327 & 3.0 & -26.0 \\
\hline 58 & 1401.7 & & & & 57.5 & 1380.7 & 58 & 1378 & 2.7 & -23.7 \\
\hline 60 & 1449.4 & & & & 59.5 & 1432.5 & & & & \\
\hline
\end{tabular}


TABLE II. Quartet of superdeformed bands in ${ }^{146-148} \mathrm{Gd}$. Spin assignments are from [12], except for a shift of two units of angular momentum in the bands ${ }^{147} \mathrm{Gd}(2 \mathrm{~b})$ and ${ }^{146} \mathrm{Gd}(1 \mathrm{~b})$. All energies are in $\mathrm{keV}$.

\begin{tabular}{|c|c|c|c|c|c|c|c|c|c|c|}
\hline \multicolumn{2}{|c|}{${ }^{148} \mathrm{Gd}(1 \mathrm{~b})$} & \multicolumn{2}{|c|}{${ }^{147} \mathrm{Gd}(1 \mathrm{~b})$} & \multicolumn{3}{|c|}{${ }^{147} \mathrm{Gd}(2 \mathrm{~b})$} & \multicolumn{2}{|c|}{${ }^{146} \mathrm{Gd}(1 \mathrm{~b})$} & \multirow[b]{2}{*}{$\Delta E_{\gamma 12}$} & \multirow[b]{2}{*}{$\Delta E_{\gamma 20}$} \\
\hline $\bar{L}$ & $E_{\gamma 0}$ & $J$ & $E_{\gamma 1}$ & $\Delta E_{\gamma 01}$ & $J$ & $E_{\gamma 1}$ & $L$ & $E_{\gamma 2}$ & & \\
\hline$\overline{26}$ & 700 & 26.5 & 696.3 & 3.7 & 25.5 & 731.2 & & & & \\
\hline 28 & 747 & 28.5 & 745.7 & 1.3 & 27.5 & 779.1 & & & & \\
\hline 30 & 797 & 30.5 & 795.4 & 1.6 & 29.5 & 826.9 & 30 & 826.7 & 0.2 & 29.7 \\
\hline 32 & 847 & 32.5 & 846.7 & 0.3 & 31.5 & 877.2 & 32 & 878.5 & -1.3 & 29.5 \\
\hline 34 & 898 & 34.5 & 899.9 & -1.9 & 33.5 & 928.6 & 34 & 929.4 & -0.8 & 31.4 \\
\hline 36 & 950 & 36.5 & 954.4 & -4.4 & 35.5 & 981.5 & 36 & 983.1 & -1.6 & 33.1 \\
\hline 38 & 1004 & 38.5 & 1009.2 & -5.2 & 37.5 & 1035.3 & 38 & 1038.8 & -3.5 & 34.8 \\
\hline 40 & 1058 & 40.5 & 1065.3 & -7.3 & 39.5 & 1090.3 & 40 & 1093.9 & -3.6 & 35.9 \\
\hline 42 & 1114 & 42.5 & 1120.4 & -6.4 & 41.5 & 1146.4 & 42 & 1148.7 & -2.3 & 34.7 \\
\hline 44 & 1171 & 44.5 & 1175.2 & -4.2 & 43.5 & 1203.1 & 44 & 1201.4 & 1.7 & 30.4 \\
\hline 46 & 1228 & 46.5 & 1228.6 & -0.6 & 45.5 & 1261.0 & 46 & 1250.3 & 10.7 & 22.3 \\
\hline 48 & 1285 & 48.5 & 1277.0 & 8.0 & 47.5 & 1319.0 & 48 & 1297.4 & 21.6 & 12.4 \\
\hline 50 & 1344 & 50.5 & 1323.3 & 20.7 & 49.5 & 1378.6 & 50 & 1345.7 & 32.9 & 1.7 \\
\hline 52 & 1403 & 52.5 & 1367.1 & 35.9 & 51.5 & 1438.8 & 52 & 1397.0 & 41.8 & -6.0 \\
\hline 54 & 1462 & 54.5 & 1413.7 & 48.3 & 53.5 & 1500.0 & 54 & 1448.3 & 51.7 & -13.7 \\
\hline 56 & 1520 & 56.5 & 1463.0 & 57.0 & 55.5 & 1559.0 & & & & \\
\hline 58 & 1580 & 58.5 & 1516.0 & 64.0 & & & & & & \\
\hline
\end{tabular}


TABLE III. Suggested quartet classification of superdeformed bands in the isotopes $Z=80$ for $A=192$ to 194 . Spin assignments are made to fulfill SSQM constraints. All energies are in keV.

\begin{tabular}{rrrrrrrrrrr}
\hline \hline \multicolumn{1}{c}{${ }^{192} \mathrm{Hg}$} & \multicolumn{7}{c}{${ }^{193} \mathrm{Hg}(2)$} & \multicolumn{7}{c}{${ }^{193} \mathrm{Hg}(3)$} & ${ }^{194} \mathrm{Hg}(3)$ & & \\
\hline$L$ & $E_{\gamma 0}$ & $J$ & $E_{\gamma 1}$ & $\Delta E_{\gamma 01}$ & $J$ & $E_{\gamma 1}$ & $L$ & $E_{\gamma 2}$ & $\Delta E_{\gamma 12}$ & $\Delta E_{\gamma 20}$ \\
\hline 10 & 214.6 & & & & 9.5 & 233.7 & & & & \\
12 & 257.7 & 12.5 & 254.3 & 3.4 & 11.5 & 274.6 & 12 & 262.3 & 12.3 & -4.6 \\
14 & 299.9 & 14.5 & 294.9 & 5.0 & 13.5 & 314.7 & 14 & 302.5 & 12.2 & -2.6 \\
16 & 341.1 & 16.5 & 334.2 & 6.9 & 15.5 & 353.5 & 16 & 342.8 & 10.7 & -1.7 \\
18 & 381.6 & 18.5 & 374.2 & 7.4 & 17.5 & 392.7 & 18 & 382.1 & 10.6 & -0.5 \\
20 & 420.8 & 20.5 & 412.9 & 7.9 & 19.5 & 431.5 & 20 & 420.4 & 11.1 & 0.4 \\
22 & 459.1 & 22.5 & 451.0 & 8.1 & 21.5 & 468.7 & 22 & 458.3 & 10.4 & 0.8 \\
24 & 496.3 & 24.5 & 488.1 & 8.2 & 23.5 & 504.7 & 24 & 494.6 & 10.1 & 1.7 \\
26 & 532.4 & 26.5 & 524.9 & 7.5 & 25.5 & 540.7 & 26 & 531.6 & 9.1 & 0.8 \\
28 & 567.9 & 28.5 & 559.9 & 8.0 & 27.5 & 575.4 & 28 & 566.4 & 9.0 & 1.5 \\
30 & 602.3 & 30.5 & 595.0 & 7.3 & 29.5 & 611.0 & 30 & 600.9 & 10.1 & 1.4 \\
32 & 635.8 & 32.5 & 628.6 & 7.2 & 31.5 & 645.0 & 32 & 635.1 & 9.9 & 0.7 \\
34 & 668.6 & 34.5 & 661.6 & 7.0 & & & 34 & 668.0 & & 0.6 \\
36 & 700.6 & 36.5 & 694.5 & 6.1 & & & 36 & 700.4 & & 0.2 \\
38 & 732.1 & 38.5 & 726.3 & 5.8 & & & 38 & 732.2 & & -0.1 \\
40 & 762.8 & 40.5 & 756.6 & 6.2 & & & 40 & 762.7 & & 0.1 \\
42 & 793.4 & & & & & & & & \\
\hline \hline
\end{tabular}

